\title{
LH-Moments of Some Distributions Useful in Hydrology
}

\author{
Md. Sharwar Murshed ${ }^{a}$, Byung-Jun Park ${ }^{b}$, Bo-Yoon Jeong ${ }^{a}$, Jeong-Soo Park ${ }^{1, a}$ \\ ${ }^{a}$ Department of Statistics, Chonnam National University \\ ${ }^{b}$ Department of Computer and Statistics, Chosun University
}

\begin{abstract}
It is already known from the previous study that flood seems to have heavier tail. Therefore, to make prediction of future extreme label, some agreement of tail behavior of extreme data is highly required. The LH-moments estimation method, the generalized form of $L$-moments is an useful method of characterizing the upper part of the distribution. LH-moments are based on linear combination of higher order statistics. In this study, we have formulated LH-moments of five distributions useful in hydrology such as, two types of three parameter kappa distributions, beta- $\kappa$ distribution, beta-p distribution and a generalized Gumbel distribution. Using LH-moments reduces the undue influences that small sample may have on the estimation of large return period events.
\end{abstract}

Keywords: Beta- $\kappa$ distribution, beta- $p$ distribution, generalized Gumbel distribution, $L$-moment, probability weighted moment, three parameter kappa distribution.

\section{Introduction}

Over the past few decades, main concern goes to characterize the extreme events such as heavy rainfall, maximum wind speed, snowfall, earth quake due to their destructive nature for human life and wealth (Coles, 2001; Park and Jung, 2002). Therefore, to judge the closeness of an observed sample to a postulated distribution, sample moment statistics, particularly, skewness and kurtosis were used previously. But these statistics were found insignificant to anticipating heavy return events, specially for small or moderate sample (Hosking and Wallis, 1997). So, an alternative approach was introduced by Hosking (1990) as an product moments for characterizing distributions and data, which are known as $L$-moments. These $L$-moments are analogous to the conventional moments but can be estimated by linear combinations of the elements of an ordered sample that is, by $L$-statistics (Hosking and Wallis, 1997).

In recent hydrological practice, the application of $L$-moments are become the most frequently used tool for predicting large return period events since 1990 due to its advantage over conventional moments (Hosking, 1990). And according to Hosking and Wallis (1997), the method of $L$-moment estimation is also less subject to bias, especially for small to moderate sample size, compared to the maximum likelihood method. Naturally, in statistical analysis of extreme events, it is often conducted of predicting large return period events therefore, more relevant to the analysis are the upper part of the distribution but product moments and moment ratios are being highly sensitive to the upper part of the distributions. To overcome this problem, Wang (1997) has introduced LH-moments(Higher order $L$-moments). LH-moments is the generalized form of $L$-moments which characterizes the upper part

This work was funded by the Korea Meteorological Administration Research and Development Program under Grant CATER 2009-4507.

${ }^{1}$ Corresponding author: Professor, Department of statistics, Chonnam National University, Gwangju 500 757 , South Korea. E-mail: jspark@jnu.ac.kr 
of the distributions and larger events in data. LH-moments calculation intentionally ignore the lower part of the data due to give more emphasis on upper part of the data for anticipating future extreme label. Recently, LH-moments estimation method has become the most popular method in hydrology (Meshgi and Khalili, 2009; Lee and Maeng, 2003).

In this study, we have formulated LH-moments for five distributions useful in hydrology such as Mielke-Johnson's three parameter kappa distribution(K3D), three parameter kappa type-II distribution (K3D-II), beta- $\kappa$ distribution, beta- $p$ distribution and a generalized Gumbel distribution(GGD).

\section{Distributions and Quantile Functions}

\subsection{Mielke-Johnson's kappa distribution}

The kappa distribution, a family of positively skewed distribution was introduce by Mielke (1973) and Mielke and Johnson (1973). It has received a good attention from hydrological point of view such as, stream flow of a river, rainfall data. The three parameter kappa distribution(K3D) was introduced by Mielke and Johnson (1974). The probability density(pdf), the cumulative distribution(cdf) and the quantile function of $\mathrm{K} 3 \mathrm{D}$ are given as, for $x \geq 0, \alpha, \beta, \theta>0$,

$$
\begin{aligned}
& f(x)=\left(\frac{\alpha \theta}{\beta}\right)\left(\frac{x}{\beta}\right)^{\theta-1}\left(\alpha+\frac{x}{\beta}\right)^{\alpha+\frac{1}{\alpha}}, \\
& F(x)=\left(\frac{x}{\beta}\right)^{\alpha \theta}\left\{\alpha+\left(\frac{x}{\beta}\right)^{\alpha \theta}\right\}^{-\frac{1}{\alpha}}, \\
& x(F)=\beta\left(\frac{\alpha F^{\alpha}}{1-F^{\alpha}}\right)^{\frac{1}{\alpha \theta}}, \quad 0<F<1,
\end{aligned}
$$

respectively. The $\mathrm{K} 3 \mathrm{D}$ is characterized by the three parameters $\alpha, \beta$ and $\theta$. Where, $\beta$ is scale and $\alpha, \theta$ are the shape parameters. When $\theta=1$ the K3D is changed to two parameter kappa distribution (K2D; Mielke, 1973; Oh et al., 2007). In this case, $\alpha$ is the only shape parameter.

\subsection{Three parameter kappa type-II distribution}

Another type of three parameter kappa distribution(K3D-II) was suggested by Park et al. (2009) which is not the same as that of Mielke and Johnson (1974). In this distribution, a new location parameter $\mu$ is introduced to the K2D. The $p d f$, $c d f$ and the quantile function of K3D-II are given as, for $x>0, \alpha, \beta>0, \mu \leq \min _{1 \leq i \leq n}\left(x_{i}\right)$,

$$
\begin{aligned}
& f(x)=\left(\frac{\alpha}{\beta}\right)\left\{\alpha+\left(\frac{x-\mu}{\beta}\right)^{\alpha}\right\}^{-\frac{\alpha+1}{\alpha}}, \\
& F(x)=\left(\frac{x-\mu}{\beta}\right)\left\{\alpha+\left(\frac{x-\mu}{\beta}\right)^{\alpha}\right\}^{-\frac{1}{\alpha}}, \\
& x(F)=\mu+\beta\left(\frac{\alpha F^{\alpha}}{1-F^{\alpha}}\right)^{\frac{1}{\alpha}}, \quad 0<F<1,
\end{aligned}
$$

respectively. Where $\mu, \alpha$ and $\beta$ are the location, shape and scale parameters respectively.

Mielke-Johnson's K3D and K3D-II are special cases of a four parameter kappa distribution (Hosking, 1994; Park and Kim, 2007). 


\subsection{Beta- $k$ distribution}

The beta- $\kappa$ distribution is the reparameterized version of a kappa distribution (Mielke and Johnson, 1974) which has been used for testing changes in extreme rainfall events (Mason et al., 1999). The $p d f, c d f$ and quantile function of beta- $\kappa$ distribution are respectively, for $x>0, \alpha, \beta, \theta>0$,

$$
\begin{aligned}
& f(x)=\frac{\alpha \theta}{\beta}\left(\frac{x}{\beta}\right)^{\alpha \theta-1}\left\{1+\left(\frac{x}{\beta}\right)^{\theta}\right\}^{-(\alpha+1)}, \\
& F(x)=\left(\frac{x}{\beta}\right)^{\theta}\left\{1+\left(\frac{x}{\beta}\right)^{\theta}\right\}^{-\alpha}, \\
& x(F)=\beta\left(\frac{F^{\frac{1}{\alpha}}}{1-F^{\frac{1}{\alpha}}}\right)^{\frac{l}{\theta}}, \quad 0<F<1 .
\end{aligned}
$$

\subsection{Beta- $p$ distribution}

The $p d f$ and $c d f$ of beta- $p$ distribution (Meilke and Johnson, 1974) are

$$
\begin{aligned}
& f(x)=\left(\frac{\alpha \theta}{\beta}\right)\left(\frac{x}{\beta}\right)^{\theta-1}\left\{1+\left(\frac{x}{\beta}\right)^{\theta}\right\}^{-(\alpha+1)}, \quad x>0, \\
& F(x)=1-\left\{1+\left(\frac{x}{\beta}\right)^{\theta}\right\}^{-\alpha}, \quad x \geq 0,
\end{aligned}
$$

respectively, where $0<\alpha, 0<\beta, 0<\theta$. The quantile function is

$$
x(F)=\beta\left\{(1-F)^{-\frac{1}{\alpha}}-1\right\}^{\frac{1}{\theta}}, \quad 0<F<1 .
$$

The beta- $p$ distribution is also known as a special case of Pareto-type distribution (Johnson and Kotz, 1970).

The beta- $\kappa$ and beta- $p$ distributions are specific restricted versions of the generalized beta distributions of the second kind. Wilks (1993) examined the performances of nine three parameter distributions to the 13 annual extreme and partial duration data sets of United States (Öztekin, 2007). Wilks (1993) found that the beta- $k$ distribution best described the extreme right tail of annual extreme series, and the beta- $p$ distribution was best for the partial duration data.

\subsection{A generalized Gumbel distribution}

A generalized Gumbel distribution(GGD) is a special case $(k=0)$ of a four parameter kappa distribution (Hosking, 1994). This distribution has not been investigated by researchers. The $p d f, c d f$ and quantile function of a GGD (Jeong, 2009) are, for $x>0$ and $\alpha>0$,

$$
\begin{aligned}
& f(x)=\frac{1}{\alpha} \exp \left(-\frac{x-\xi}{\alpha}\right) F(x)^{1-h}, \\
& F(x)=\left\{1-h \exp \left(-\frac{x-\xi}{\alpha}\right)\right\}^{\frac{1}{h}},
\end{aligned}
$$




$$
x(F)= \begin{cases}\xi-\alpha \log \left(\frac{1-F^{h}}{h}\right), & \text { if } h>0, \\ \xi+\alpha \log \left(\frac{-h}{F^{h}-1}\right), & \text { if } h<0,\end{cases}
$$

for $0<F<1$, where $\xi, \alpha$ and $h$ are the parameters of location, scale and shape respectively, and under the restriction $\xi+\alpha \log h \leq \min _{1 \leq i \leq n}\left(x_{i}\right)$ for $h>0$. When $h<0$ the $c d f$ (2.14) is also exist without any restrictions. When $h \rightarrow 0$, the $p d f$ of GGD changes to the $p d f$ of Gumbel distribution. More details on GGD are available in Jeong (2009).

\section{LH-Moments}

For a given sample of size $m$ drawn from a distribution $F(x)=\operatorname{Pr}(X \leq x)$, the expectation of an order statistic can be written as

$$
E\left[X_{j: m}\right]=\frac{m !}{(j-1) !(m-j) !} \int_{0}^{1} x(F) F^{j-1}(1-F)^{m-j} d F .
$$

The first few $L H$-moments(Higher order $L$-moments) are defined by Wang (1997) for $\eta=0,1,2, \ldots$

$$
\begin{aligned}
& \lambda_{1}^{\eta}=E\left[X_{(\eta+1):(\eta+1)}\right], \\
& \lambda_{2}^{\eta}=\frac{1}{2} E\left[X_{(\eta+2):(\eta+2)}-X_{(\eta+1):(\eta+2)}\right], \\
& \lambda_{3}^{\eta}=\frac{1}{3} E\left[X_{(\eta+3):(\eta+3)}-2 X_{(\eta+2):(\eta+3)}+X_{(\eta+1):(\eta+3)}\right], \\
& \lambda_{4}^{\eta}=\frac{1}{4} E\left[X_{(\eta+4):(\eta+4)}-3 X_{(\eta+3):(\eta+4)}+3 X_{(\eta+2):(\eta+4)}-X_{(\eta+1):(\eta+4)}\right] .
\end{aligned}
$$

According to Wang (1997), $\lambda_{1}^{\eta}$ is denoted as the expectation of the largest variable in a sample of size $(\eta+1)$, which is a measure of location of a distribution; $\lambda_{2}^{\eta}$ is the one half times the difference between the largest and the second largest variables in a sample of size $(\eta+2)$, characterizes the spreadness of the upper part of a distribution; $\lambda_{3}^{\eta}$ is considering the expectations of the largest three variables in a sample of size $(\eta+3)$; and $\lambda_{4}^{\eta}$ provides the measure of pickness of the upper part of a distribution of the largest four variables in a sample of size $(\eta+4)$. LH-moments are also called $L 1$ moments, $L 2$ moments and so on respectively. When $\eta=0$, LH-moments become identical to Hosking's (1990) $L$-moments. As $\eta$ increases, LH-moments reflects more and more characteristics of the upper part of the distribution (Wang, 1997).

Let us suppose, $X_{(1)}, X_{(2)}, \ldots, X_{(n)}$ be the ordered sample, the following equation can produce the $\eta^{\text {th }}$ order sample LH-moments (Wang, 1997);

$$
\begin{aligned}
& \hat{\lambda}_{1}^{\eta}=\frac{1}{\left(\begin{array}{c}
n \\
\eta+1
\end{array}\right)} \sum_{i=1}^{n}\left(\begin{array}{c}
i-1 \\
\eta
\end{array}\right) X_{(i)}, \\
& \hat{\lambda}_{2}^{\eta}=\frac{1}{2} \frac{1}{\left(\begin{array}{c}
n \\
\eta+2
\end{array}\right)} \sum_{i=1}^{n}\left[\left(\begin{array}{c}
i-1 \\
\eta+1
\end{array}\right)-\left(\begin{array}{c}
i-1 \\
\eta
\end{array}\right)\left(\begin{array}{c}
n-i \\
1
\end{array}\right)\right] X_{(i)}, \\
& \hat{\lambda}_{3}^{\eta}=\frac{1}{3} \frac{1}{\left(\begin{array}{c}
n \\
\eta+3
\end{array}\right)} \sum_{i=1}^{n}\left[\left(\begin{array}{l}
i-1 \\
\eta+2
\end{array}\right)-2\left(\begin{array}{c}
i-1 \\
\eta+1
\end{array}\right)\left(\begin{array}{c}
n-i \\
1
\end{array}\right)+\left(\begin{array}{c}
i-1 \\
\eta
\end{array}\right)\left(\begin{array}{c}
n-i \\
2
\end{array}\right)\right] X_{(i)},
\end{aligned}
$$


where $\left(\begin{array}{c}m \\ j\end{array}\right)=m ! / j !(m-j) !$ and is equal to zero when $j>m$.

\section{Probability Weighted Moments, L- and LH-Moments}

For the efficient calculation of LH-moments, the so-called probability weighted moments(PWMs) are described. Suppose $X$ be a real valued random variable with distribution function $F$. According to Greenwood et al. (1979), the PWMs of $X$ is defined by

$$
M_{p, r, s}=E\left[X^{p}\{F(X)\}^{r}\{1-F(X)\}^{s}\right]=\int x^{p}\{F(x)\}^{r}\{1-F(x)\}^{s} d F(x),
$$

where, $p, r$ and $s$ are real numbers. The above definition of PWMs is valid both for continuous and discrete random variables. For the quantile function $x(F)$, we have

$$
M_{p, r, s}=\int_{0}^{1}\{x(F)\}^{p} F^{r}(1-F)^{s} d F .
$$

One approach of the PWM is to work with $M_{p, 0,0}, p=1,2, \ldots$, which are the conventional noncentral moments of $X$. We will instead work with the moments $M_{1, r, s}$ into which $X$ enters linearly and in particular with the quantities

$$
\begin{aligned}
\boldsymbol{\alpha}_{r} & =M_{1,0, r}=E\left[X\{1-F(X)\}^{r}\right], \quad r=0,1, \ldots, \\
\beta_{r} & =M_{1, r, 0}=E\left[X\{F(X)\}^{r}\right], \quad r=0,1, \ldots
\end{aligned}
$$

Historically, PWMs are the precursor of $L$ - and LH-moments. Hosking (1990) defined $L$-moments as a linear combination of PWMs:

$$
\lambda_{r+1}=(-1)^{r} \sum_{k=0}^{r} P_{r, k}^{*} \alpha_{k}=\sum_{k=0}^{r} P_{r, k}^{*} \beta_{k}, \quad \text { for } r=0,1,2, \ldots,
$$

with $P_{r, k}^{*}=(-1)^{r-k}\left(\begin{array}{l}r \\ \kappa\end{array}\right)\left(\begin{array}{c}r+\kappa \\ \kappa\end{array}\right)$, which is called as the shifted Legendre polynomials. The " $L$ " in $L$ moments emphasizes the construction of $L$-moments from linear combinations of order statistics. In (4.5), $\lambda_{1}$ is the measure of location; $\lambda_{2}$ is a measure of scale; $\lambda_{3}$ is a measure of skewness and $\lambda_{4}$ is a measure of kurtosis, respectively. The $L$-moment ratios are defined as $\tau_{2}=\lambda_{2} / \lambda_{1}, \tau_{3}=\lambda_{3} / \lambda_{2}$ and $\tau_{4}=\lambda_{4} / \lambda_{2}$ which represents $L$-CV, $L$-skewness and $L$-kurtosis respectively.

The relation between normalized PWMs and LH-moments are useful to derive population LHmoments of those distribution which PWMs are already exits (Wang, 1997). The normalized PWMs are defined as in two forms:

$$
\begin{aligned}
B_{r} & =\int_{0}^{1} x(F) F^{r} d F / \int_{0}^{1} F^{r} d F \\
& =(r+1) \int_{0}^{1} x(F) F^{r} d F=(r+1) \beta_{r}, \\
A_{r} & =\int_{0}^{1} x(F)(1-F)^{r} d F / \int_{0}^{1} F^{r} d F \\
& =(r+1) \int_{0}^{1} x(F)(1-F)^{r} d F=(r+1) \alpha_{r},
\end{aligned}
$$


where $\beta_{r}$ and $\alpha_{r}$, given in (4.3) and (4.4), are called as the standard PWMs (Greenwood et al., 1979). Now substituting (3.1) into (3.2) (3.5) yield useful relation between LH-moments and normalized PWMs:

$$
\begin{aligned}
\lambda_{1}^{\eta}= & B_{\eta}, \quad \lambda_{2}^{\eta}=\frac{1}{2 !}(\eta+2)\left[B_{\eta+1}-B_{\eta}\right], \\
\lambda_{3}^{\eta}= & \frac{(\eta+3)}{3 !}\left[(\eta+4) B_{\eta+2}-2(\eta+3) B_{\eta+1}+(\eta+2) B_{\eta}\right], \\
\lambda_{4}^{\eta}= & \frac{(\eta+4)}{4 !}\left[(\eta+6)(\eta+5) B_{\eta+3}-3(\eta+5)(\eta+4) B_{\eta+2}\right. \\
& \left.+3(\eta+4)(\eta+3) B_{\eta+1}-(\eta+3)(\eta+2) B_{\eta}\right] .
\end{aligned}
$$

The above formula (4.8) (4.10) are actually used in deriving LH-moments(see Appendix). Here it is also noticeable that, LH-moment of order 2 or above, the sum of the coefficients on the right-hand side of the equations is always zero.

\section{LH-Moments Calculation for Some Distributions}

\subsection{Mielke-Johnson's kappa distribution}

The normalized PWMs of K3D is obtained for $r=1,2, \ldots$, under the restriction $\alpha \theta>1$,

$$
B_{r}=(r+1) \beta \alpha^{\frac{1}{\alpha \theta}-1} B\left(\frac{r \theta+\theta+1}{\alpha \theta}, \frac{\alpha \theta-1}{\alpha \theta}\right),
$$

where $B()$ is a beta function. The derivations of PWMs and first LH-moment from the distributions of Section 2.1 to 2.5 are provided in Appendix. Now substituting (5.1) into (3.2) to (3.5) yield

$$
\begin{aligned}
& \lambda_{1}^{\eta}=(\eta+1) \beta \alpha^{\frac{1}{\alpha \theta}-1} X_{1}, \\
& \lambda_{2}^{\eta}=\frac{(\eta+2) \beta \alpha^{\frac{1}{\alpha \theta}}-1}{2}\left[(\eta+2) X_{2}-(\eta+1) X_{1}\right], \\
& \lambda_{3}^{\eta}=\frac{(\eta+3) \beta \alpha^{\frac{1}{\alpha \theta}}-1}{3 !}\left[V_{4} X_{3}-2 V_{3} X_{2}+V_{2} X_{1}\right], \\
& \lambda_{4}^{\eta}=\frac{(\eta+4) \beta \alpha^{\frac{1}{\alpha \theta}}-1}{4 !}\left[W_{6} X_{4}-3 W_{5} X_{3}+3 W_{4} X_{2}-W_{3} X_{1}\right],
\end{aligned}
$$

where, $X_{k}=B((\eta \theta+k \theta+1) /(\alpha \theta),(\alpha \theta-1) /(\alpha \theta))$, for $k=1,2,3,4$, and under the restriction, $\alpha \theta>1$. Also $W_{i}=(\eta+i)(\eta+i-1)(\eta+i-2)$ for $i=3,4,5,6$ and $V_{j}=(\eta+j)(\eta+j-1)$ for $j=2,3,4$.

\subsection{Three parameter kappa type-II distribution}

The normalized PWMs of K3D-II is for $r=1,2, \ldots$, under the restriction $\alpha>1$,

$$
B_{r}=\mu+(r+1) \beta \alpha^{\frac{1}{\alpha}-1} B\left(\frac{r+2}{\alpha}, \frac{\alpha-1}{\alpha}\right) .
$$

The first four LH-moments of K3D-II are

$$
\lambda_{1}^{\eta}=\mu+(\eta+1) \beta \alpha^{\frac{1}{\alpha}-1} Y_{2},
$$




$$
\begin{aligned}
& \lambda_{2}^{\eta}=\frac{(\eta+2) \beta \alpha^{\frac{1}{\alpha}-1}}{2}\left[(\eta+2) Y_{3}-(\eta+1) Y_{2}\right], \\
& \lambda_{3}^{\eta}=\frac{(\eta+3) \beta \alpha^{\frac{1}{\varepsilon}-1}}{3 !}\left[V_{4} Y_{4}-2 V_{3} Y_{3}+V_{2} Y_{2}\right], \\
& \lambda_{4}^{\eta}=\frac{(\eta+4) \beta \alpha^{\frac{1}{r}-1}}{4 !}\left[W_{6} Y_{5}-3 W_{5} Y_{4}+3 W_{4} Y_{3}-W_{3} Y_{2}\right],
\end{aligned}
$$

where $Y_{k}=B((\eta+k) / \alpha,(\alpha-1) / \alpha)$ for $k=2,3,4,5$, under the restriction, $\alpha>1$.

\subsection{Beta- $\kappa$ distribution}

The normalized PWMs of beta- $\kappa$ distribution is, for $r=1,2, \ldots$,

$$
B_{r}=(\eta+1) \beta \alpha B\left(\frac{1}{\theta}+r \alpha+\alpha, \frac{\theta-1}{\theta}\right)
$$

under the restriction $\theta>1$. First few population LH-moments of beta- $\kappa$ distribution are obtained by

$$
\begin{aligned}
& \lambda_{1}^{\eta}=(\eta+1) \beta \alpha Z_{1}, \\
& \lambda_{2}^{\eta}=\frac{\beta \alpha(\eta+2)}{2}\left[(\eta+2) Z_{2}-(\eta+1) Z_{1}\right], \\
& \lambda_{3}^{\eta}=\frac{\alpha \beta(\eta+3)}{3 !}\left[V_{4} Z_{3}-2 V_{3} Z_{2}+V_{2} Z_{1}\right], \\
& \lambda_{4}^{\eta}=\frac{\beta \alpha(\eta+4)}{4 !}\left[W_{6} Z_{4}-3 W_{5} Z_{3}+3 W_{4} Z_{2}-3 W_{3} Z_{1}\right],
\end{aligned}
$$

under the restriction $\theta>1$, where, $Z_{k}=B(\alpha \eta+1 / \theta+k \alpha,(\theta-1) / \theta)$ for $k=1,2,3,4$.

\subsection{Beta- $p$ distribution}

The normalized PWMs of beta- $p$ distribution (Mielke and Johnson, 1974) are calculated from the second form of normalized PWM $A_{r}$ given in (4.7)(see Appendix). Its are, for $r=1,2, \ldots$,

$$
A_{r}=(r+1) \beta \alpha B\left(\alpha r-\frac{1}{\theta}+\alpha, 1+\frac{1}{\theta}\right),
$$

under the restriction $\alpha \theta>1 /(r+1)$. The LH-moments of beta- $p$ distribution are obtained as, under the restriction $\alpha \theta>1(\eta+i)$,

$$
\begin{aligned}
\lambda_{1}^{\eta} & =(\eta+1) \beta \alpha P_{1}, \\
\lambda_{2}^{\eta} & =\frac{(\eta+2) \alpha \beta}{2}\left[(\eta+1) P_{1}-(\eta+2) P_{2}\right], \\
\lambda_{3}^{\eta} & =\frac{(\eta+3) \alpha \beta}{3 !}\left[V_{2} P_{1}-2 V_{3} P_{2}+V_{4} P_{3}\right], \\
\lambda_{4}^{\eta} & =\frac{(\eta+4) \alpha \beta}{4 !}\left[W_{3} P_{1}-3 W_{4} P_{2}+3 W_{5} P_{3}-W_{6} P_{4}\right],
\end{aligned}
$$

where $P_{i}=B(\alpha \eta-1 / \theta+i \alpha,(\theta+1) / \theta)$, for $i=1,2,3,4$. 


\subsection{A generalized Gumbel distribution}

The calculation of LH-moments of a GGD are divided into two parts according to $h>0$ and $h<0$.

Case I: $h>0$.

The normalized PWMs of generalized Gumbel distribution for $r=1,2, \ldots$ are

$$
B_{r}=\xi+\alpha\left[\Psi\left(\frac{r}{h}+\frac{1}{h}+1\right)-\Psi(1)\right]+\alpha \log h,
$$

where $\Psi$ is a digamma function $(\Psi(x)=d \log \Gamma(x) / d x)$ and $\Psi(1)$ is the Euler's constant $(=0.577215665)$. The first four LH-moments of GGD for $h>0$ are,

$$
\begin{aligned}
& \lambda_{1}^{\eta}=\xi+\alpha\left[N_{0}-\Psi(1)\right]+\alpha \log h, \\
& \lambda_{2}^{\eta}=\frac{\alpha(\eta+2)}{2}\left[N_{1}-N_{0}\right], \\
& \lambda_{3}^{\eta}=\frac{\alpha(\eta+3)}{3 !}\left[(\eta+4) N_{2}-2(\eta+3) N_{1}+(\eta+2) N_{0}\right], \\
& \lambda_{4}^{\eta}=\frac{\alpha(\eta+4)}{4 !}\left[V_{6} N_{3}-3 V_{5} N_{2}+3 V_{4} N_{1}-V_{3} N_{0}\right],
\end{aligned}
$$

where $N_{i}=\Psi((\eta+i) / h+1 / h+1)$ for $i=0,1,2,3$ and $V_{j}=(\eta+j)(\eta+j-1)$ for $j=3,4,5,6$.

Case II: $h<0$.

Again the normalized PWMs of GGD for $r=1,2, \ldots$, are

$$
B_{r}=\xi+\alpha\left[\Psi\left(\frac{-r}{h}-\frac{1}{h}\right)-\Psi(1)\right]+\alpha \log (-h) .
$$

First four LH-moments for $h<0$ are given by

$$
\begin{aligned}
& \lambda_{1}^{\eta}=\xi+\alpha\left[M_{0}-\Psi(1)\right]+\alpha \log (-h), \\
& \lambda_{2}^{\eta}=\frac{\alpha(\eta+2)}{2}\left[M_{1}-M_{0}\right], \\
& \lambda_{3}^{\eta}=\frac{\alpha(\eta+3)}{3 !}\left[(\eta+4) M_{2}-2(\eta+3) M_{1}+(\eta+2) M_{0}\right], \\
& \lambda_{4}^{\eta}=\frac{\alpha(\eta+4)}{4 !}\left[V_{6} M_{3}-3 V_{5} M_{2}+3 V_{4} M_{1}-V_{3} M_{0}\right],
\end{aligned}
$$

where $M_{j}=\Psi((-\eta-j) / h-1 / h)$ for $j=0,1,2,3$.

\section{Concluding Remarks}

LH-moments are based on linear combinations of higher order statistics. In this study, we have derived the LH-moments of Mielke-Johnson's three parameter kappa distribution, three parameter kappa type-II distribution, beta-k distribution, beta- $p$ distribution and a generalized Gumbel distribution for statistical use of convenience. The distributions have been useful in hydrology, especially for the frequency analysis in which estimating the high return value is important. Parameters can be estimated by matching population LH-moments to the corresponding sample LH-moments. In future, we 
will show the parameter estimation from LH-moments of a four parameter kappa distribution (Hosking, 1994; Park and Kim, 2007) and Wakeby distribution (Park et al., 2001) which are also useful in hydrology. In addition, we will carry out the Monte Carlo study to select the best value of $\eta$ and investigate the effect of using LH-moments on high quantile estimation.

\section{Appendix: Derivation of PWMs and First LH-Moment}

\section{A.1: Mielke-Johnson's kappa distribution}

The probability weighted moments(PWMs) with the first LH-moment of Mielke-Johnson's K3D are

$$
\begin{aligned}
B_{\eta}=\lambda_{1}^{\eta} & =(\eta+1) \int_{0}^{1} x(F) F^{\eta} d F \\
& =(\eta+1) \int_{0}^{1} \beta\left(\frac{\alpha F^{\alpha}}{1-F^{\alpha}}\right)^{\frac{1}{\alpha \theta}} F^{\eta} d F \\
& \left.=(\eta+1) \beta \alpha^{\frac{1}{\alpha \theta}-1} \int_{0}^{1} Z^{\frac{\eta \theta+\theta+1}{\alpha \theta}-1}(1-Z)^{\frac{\alpha \theta-1}{\alpha \theta}-1} d Z \quad \text { (by letting, } F^{\alpha}=Z\right) \\
& =(\eta+1) \beta \alpha^{\frac{1}{\alpha \theta}-1} B\left(\frac{\eta \theta+\theta+1}{\alpha \theta}, \frac{\alpha \theta-1}{\alpha \theta}\right), \quad \alpha \theta>1 .
\end{aligned}
$$

Now substituting $r=\eta+1, \eta+2$ and $\eta+3$ into (4.6) yield $B_{\eta+1}, B_{\eta+2}$ and $B_{\eta+3}$ which will provide $\lambda_{2}^{\eta}, \lambda_{3}^{\eta}$ and $\lambda_{4}^{\eta}$ respectively.

\section{A.2: Three parameter kappa type-II distribution}

The PWMs and first LH-moment of K3D-II are

$$
\begin{aligned}
B_{\eta}=\lambda_{1}^{\eta} & =(\eta+1) \int_{0}^{1} \mu+\beta\left(\frac{\alpha F^{\alpha}}{1-F^{\alpha}}\right) F^{\eta} d F \\
& \left.=\mu+(\eta+1) \beta \alpha^{\frac{1}{\alpha}-1} \int_{0}^{1} Z^{\frac{\eta+2}{\alpha}-1}(1-Z)^{\frac{\alpha-1}{\alpha}-1} d Z \quad \text { (by letting, } F^{\alpha}=Z\right) \\
& =\mu+(\eta+1) \beta \alpha^{\frac{1}{\alpha}-1} B\left(\frac{\eta+2}{\alpha}, \frac{\alpha-1}{\alpha}\right), \quad \alpha>1 .
\end{aligned}
$$

Similarly, $\lambda_{2}^{\eta}, \lambda_{3}^{\eta}$ and $\lambda_{4}^{\eta}$ are calculated like A.1.

\section{A.3: Beta- $\kappa$ distribution}

The PWMs and the first LH-moment of beta- $\kappa$ distribution are

$$
\begin{aligned}
B_{\eta}=\lambda_{1}^{\eta} & =(\eta+1) \int_{0}^{1} \beta\left(\frac{F^{\frac{1}{\alpha}}}{1-F^{\frac{1}{\alpha}}}\right)^{\frac{1}{\theta}} F^{\eta} d F \\
& \left.=(\eta+1) \beta \alpha \int_{0}^{1} Z^{\frac{1}{\theta}+\alpha \eta+\alpha-1}(1-Z)^{\frac{\theta-1}{\theta}-1} d Z \quad \text { (by letting, } F^{\frac{1}{\alpha}}=Z\right) \\
& =(\eta+1) \beta \alpha B\left(\frac{1}{\theta}+\alpha \eta+\alpha, \frac{\theta-1}{\theta}\right), \theta>1 .
\end{aligned}
$$




\section{A.4: Beta- $p$ distribution}

To calculate PWMs and LH-moments of beta- $p$ distribution, we use the normalized PWM $A_{r}$ given in (4.7), because calculating the explicit form of $B_{r}$ is difficult. Since $\alpha_{r}$ and $\beta_{r}$ are interchangeable, the following relation between PWMs and LH-moments is derived from (4.8) (4.10):

$$
\begin{aligned}
\lambda_{1}^{\eta} & =A_{\eta}, \\
\lambda_{2}^{\eta} & =\frac{1}{2 !}(\eta+2)\left[A_{\eta}-A_{\eta+1}\right], \\
\lambda_{3}^{\eta} & =\frac{(\eta+3)}{3 !}\left[(\eta+2) A_{\eta}-2(\eta+3) A_{\eta+1}+(\eta+4) A_{\eta+2}\right], \\
\lambda_{4}^{\eta} & =\frac{(\eta+4)}{4 !}\left[(\eta+3)(\eta+2) A_{\eta}-3(\eta+4)(\eta+3) A_{\eta+1}+3(\eta+5)(\eta+4) A_{\eta+2}-(\eta+6)(\eta+5) A_{\eta+3}\right] .
\end{aligned}
$$

Using the above relations, the PWM and the first LH-moment of beta- $p$ distribution are

$$
\begin{aligned}
A_{\eta}=\lambda_{1}^{\eta} & =(\eta+1) \int_{0}^{1} x(F)(1-F)^{\eta} d F \\
& =(\eta+1) \int_{0}^{1} \beta\left[\frac{1}{(1-F)^{\frac{1}{\alpha}}}-1\right]^{\frac{1}{\theta}}(1-F)^{\eta} d F \\
& \left.=(\eta+1) \beta \alpha \int_{0}^{1} Z^{\eta \alpha-\frac{1}{\theta}+\alpha-1}(1-Z)^{\frac{\theta+1}{\theta}-1} d Z \quad \text { [by letting, }(1-F)^{\frac{1}{\alpha}}=Z\right] \\
& =(\eta+1) \beta \alpha B\left(\alpha \eta-\frac{1}{\theta}+\alpha, 1+\frac{1}{\theta}\right), \quad \alpha \theta>\frac{1}{\eta+1} .
\end{aligned}
$$

$\lambda_{2}^{\eta}, \lambda_{3}^{\eta}$ and $\lambda_{4}^{\eta}$ are calculated in similar way like A.1.

\section{A.5: A generalized Gumbel distribution}

Case I: $h>0$.

The PWMs of a GGD are

$$
\begin{aligned}
B_{\eta}=\lambda_{1}^{\eta} & =(\eta+1) \int_{0}^{1}\left[\xi-\alpha \log \left(\frac{1-F^{h}}{h}\right)\right] F^{\eta} d F \\
& =\xi-\alpha(\eta+1) \int_{0}^{1} \log \left(1-F^{h}\right) F^{\eta} d F+\alpha \log h \\
& \left.=\xi-\frac{\alpha(\eta+1)}{h} \int_{0}^{1} u^{\frac{\eta+1}{h}-1} \log (1-u) d u+\alpha \log h \quad \text { [by letting } F^{h}=u\right] \\
& =\xi+\alpha\left[\Psi\left(\frac{\eta+1}{h}+1\right)-\Psi(1)\right]+\alpha \log h,
\end{aligned}
$$

$\lambda_{2}^{\eta}, \lambda_{3}^{\eta}$ and $\lambda_{4}^{\eta}$ can be obtained in similar way like A.1.

Case II: $h<0$. 
The PWMs with first LH-moment of GGD are

$$
\begin{aligned}
B_{\eta}=\lambda_{1}^{\eta} & =(\eta+1) \int_{0}^{1}\left[\xi+\alpha \log \left(\frac{-h}{F^{h}-1}\right)\right] F^{\eta} d F \\
& =\xi-\frac{(\eta+1) \alpha}{h}[A+B-C]
\end{aligned}
$$

where,

$$
\begin{array}{ll}
A=\int_{0}^{1} \log (-h) u^{-\frac{\eta}{h}-\frac{1}{h}-1} d u, & \text { [by letting, } \left.F^{-h}=u\right] \\
B=\int_{0}^{1}(\log u) u^{-\frac{\eta}{h}-\frac{1}{h}-1} d u, & C=\int_{0}^{1} \log (1-u) u^{-\frac{\eta}{h}-\frac{1}{h}-1} d u .
\end{array}
$$

Now equating A, B and C by using the following formula (Gradshteyn and Ryzhik, 1980)(p. 538 and 558):

$$
\begin{aligned}
\int_{0}^{1} x^{\mu-1} \ln x\left(1-x^{r}\right)^{\gamma-1} d x & =\frac{1}{r^{2}} B\left(\frac{\mu}{r}, v\right)\left[\Psi\left(\frac{\mu}{r}\right)-\Psi\left(\frac{\mu}{r}+v\right)\right], \\
\int_{0}^{1} x^{\mu-1} \ln (1-x) d x & =-\frac{1}{\mu}[\Psi(\mu+1)-\Psi(1)],
\end{aligned}
$$

where $\Psi$ is a digamma function, we have the first LH-moment of GGD for $h<0$;

$$
\lambda_{1}^{\eta}=\xi+\alpha\left[\Psi\left(-\frac{\eta}{h}-\frac{1}{h}\right)-\Psi(1)\right]+\alpha \log (-h) .
$$

$\lambda_{2}^{\eta}, \lambda_{3}^{\eta}$ and $\lambda_{4}^{\eta}$ are calculated in a similar way.

\section{References}

Coles, S. (2001). An Introduction to Statistical Modeling of Extreme Values, Springer-Verlag, London. Gradshteyn, I. S. and Ryzhik, M. (1980). Table of Integrals, Series and Products, 4th ed., Academic Press, New York.

Greenwood, J. A., Landwehr, J. M., Matalas, N. C. and Wallis, J. R. (1979). Probability weighted moments: Definition and relation to parameters of distribution expressible in inverse form, Water Resources Research, 15, 1049-1054.

Hosking, J. R. M. (1990), $L$-moments: Analysis and estimation of distributions using linear combinations of order statistics, Journal of Royal Statistical Society, Series B, 52, 105-124.

Hosking, J. R. M. (1994). The four-parameter Kappa distribution, IBM Journal of Research and Development, 38, 251-258.

Hosking, J. R. M. and Wallis J. R. (1997). Regional Frequency Analysis: An Approach Based on L-moments, Cambridge University Press, Cambridge, UK.

Jeong, B. Y. (2009). On the Properties of a Generalized Gumbel Distribution and r-kappa Distribution, Ph.D. Thesis, Chonnam National University, Gwangju.

Johnson, N. L. and Kotz, S. (1970). Continuous Univariate Distributions-1, Wiley Interscience, New York. 
Lee, S. H. and Maeng, S. J. (2003). Comparison and analysis of design floods by the change in the order of LH-moment methods, Irrigation and Drainage, 52, 231-245.

Mason, S. J., Waylen, P. R., Mimmack, G. M., Rajaratnam, B. and Harrison, J. M. (1999). Changes in extreme rainfall events in South Africa, Climatic Change, 41, 249-257.

Meshgi, A. and Khalili, D. (2009). Comprehensive evaluation of regional flood frequency analysis by L- and LH-moments. I. A re-visit to regional homogeneity, Stochastic Environmental Research and Risk Assessment, 23, 119-135.

Mielke, P. W. (1973). Another family of distributions for describing and analyzing precipitation data, Journal of Applied Meteorology, 12, 275-280.

Mielke, P. W. and Johnson, E. S. (1973). Three-parameter kappa distribution maximum likelihood estimates and likelihood ratio tests, Monthly Weather Review, 101, 701-711.

Mielke, P. W. and Johnson, E. S. (1974). Some generalized beta distributions of the second kind having desirable application features in hydrology and meteorology, Water Resources Research, 10, 223-226.

Oh, M., Kim, S., Park, J. S. and Son, Y. S. (2007). Bayesian estimation of the two-parameter kappa distribution, Communications of the Korean Statistical Society, 14, 355-363.

Öztekin, T. (2007). Wakeby distribution for representing annual extreme and partial duration rainfall series, Meteorological Applications, 14, 381-387.

Park, J. S., Jung, H. S., Kim, R. S. and Oh, J. H. (2001). Modelling summer extreme rainfall over the Korean peninsula using Wakeby distribution, International Journal of Climatology, 21, 13711384.

Park, J. S. and Jung, H. S. (2002). Modelling Korean extreme rainfall using a Kappa distribution and maximum likelihood estimate, Theoretical and Applied Climatology, 72, 55-64.

Park, J. S. and Kim, T. Y. (2007). Fisher information matrix for a four-parameter Kappa distribution, Statistics \& Probability Letters, 77, 1459-1466.

Park, J. S., Seo, S. C. and Kim, T. Y. (2009). A kappa distribution with a hydrological application, Stochastic Environmental Research and Risk Assessment, 23, 579-586.

Wang, Q. J. (1997). LH moments of statistical analysis of extreme events, Water Resources Research, 33, 2841-2848.

Wilks, D. S. (1993). Comparison of three-parameter probability distributions for representing annual extreme and partial duration precipitation series, Water Resources Research, 29, 3543-3549. 